\title{
Per- und subtrochantäre Femurfrakturen
}

\author{
Andreas Luther, Steffen Langenhan, Karl Heinrich Winker
}

\section{Zusammenfassung}

Die demographische Entwicklung bedingt eine Zunahme der Inzidenz proximaler Oberschenkelfrakturen. Der verletzte alte Mensch ist vital bedroht. Es erwächst der Anspruch auf eine rasche notfallmäßige Versorgung der Fraktur. Nur geringe Abweichungen von einer anatomischen Reposition beeinträchtigen die erforderliche Primärstabilität und führen im osteoporotisch veränderten Knochen zu intra- und postoperativen Komplikationen. Vorgestellt werden Lösungsmöglichkeiten nach fehlgeschlagenen Osteosynthesen per- und subtrochantärer Femurfrakturen. Da diese Lösungen nicht immer zu einem befriedigenden Ergebnis führen, der alte Patient einen Zweiteingriff wenig toleriert, sollen Hinweise und Tricks zur Erzielung einer primär belastungsstabilen Situation auch bei Problemfrakturen aufgezeigt werden.

Die vermeintlich einfache Anwendung besonders der intramedullären Implantate [2], das minimalinvasive Vorgehen und die hohe Inzidenz verleiten zur Bagatellisierung der Verletzung. Werden jedoch bei der Stabilisierung der instabilen pertrochantären Femurfraktur (AO-Klassifikation 31A2) nur geringe Abweichungen von der anatomischen Reposition toleriert, muss im osteoporotisch veränderten Knochen eine intra- oder postoperative Komplikation befürchtet werden. Selbst nach nur einem Eingriff wird die Sterberate während des Klinikaufenthalts in der Literatur mit bis zu $4 \%$ angegeben [10].

Da ein Zweiteingriff vom alten Menschen nur schlecht toleriert wird, die Ergebnisse der Salvage Procedures häufig unbefriedigend sind, erscheint es besonders wichtig, auf Erfahrungen und Tricks zur Erzielung einer primär belastungsstabilen Situation hinzuweisen [4].

Insbesondere an die Versorgung der instabilen pertrochantären Femurfraktur sind prä- und intraoperativ höchste Ansprüche zu stellen!

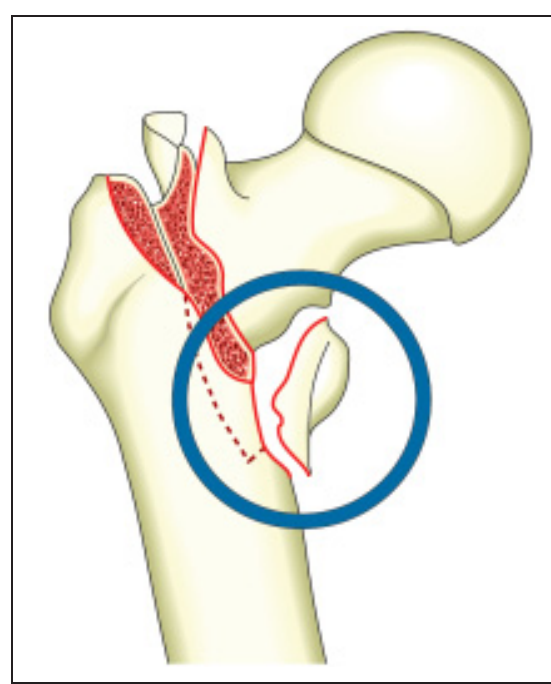

Abb.1 Instabile pertrochantäre Femurfraktur (AO-Klassifikation 31A2).

OP-JOURNAL 2004; 20: 142-149

(c) Georg Thieme Verlag KG Stuttgart · New York



Abb. 2 Korrekturverlust und Cut-out nach Stabilisierung einer A2-Fraktur mit DHS und Trochanterabstützplatte.

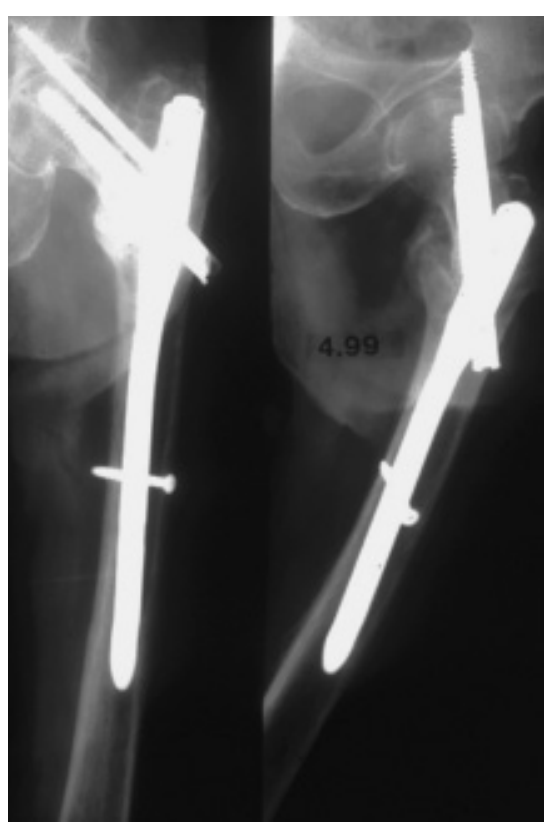

Abb. 3 Z-Effekt zwischen Antirotations- und Schenkelhalsschraube des PFN. 


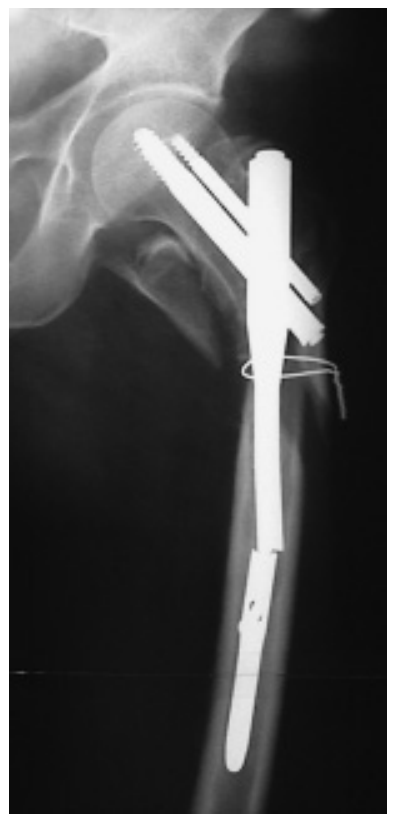

Abb. 4 Materialbruch eines PFN als Ausdruck der Instabilität nach Osteosynthese einer A2Fraktur.

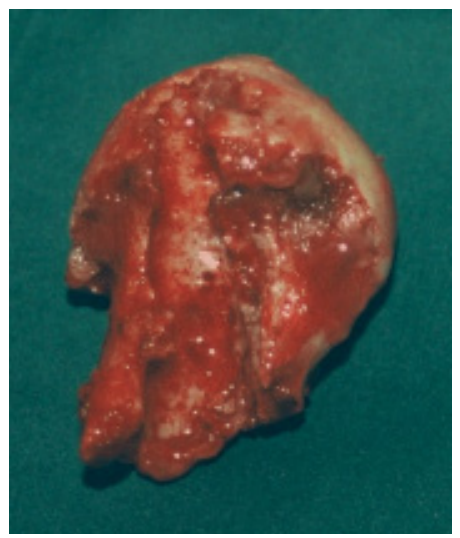

Abb. 5 Hüftkopfnekrose nach fehlgeschlagener Osteosynthese.

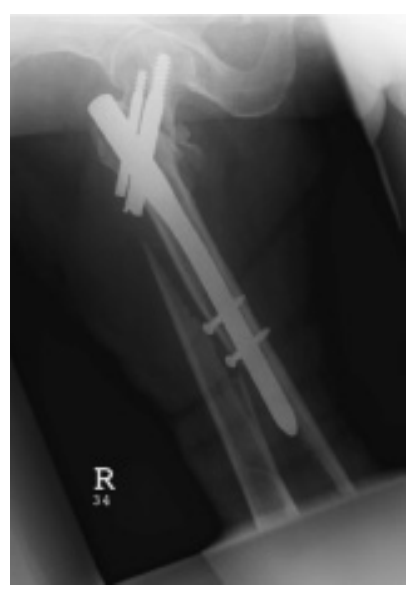

Abb. 6 Periimplantäre Fraktur bei einliegendem PFN.

\section{Instabile pertrochantäre Femurfrakturen}

Stabile pertrochantäre Femurfrakturen können mit einem extra- oder intramedullären Implantat sicher stabilisiert werden $[3,8]$. Implantat der Wahl zur Versorgung der instabilen pertrochantären Femurfraktur (Abb.1) ist ein intramedullärer Kraftträger mit Hüftkomponente (PFN, Gammanagel, Classic Nail und andere).

Die Komplikationsrate wird in der Literatur mit bis zu $14 \%$ angegeben $[1,4,7,9)$.

Folgende Komplikationen treten auf:

- Korrekturverlust (Abb.2)

- Cut-out (Abb.2)

- Z-Effekt (Abb.3)

- Implantatbruch (Abb.4)

- Hüftkopfnekrose (Abb.5)

- Periimplantäre Fraktur (Abb.6).

Die Abb. 7 und 8a,b zeigen ein zunächst tolerables Ergebnis nach Versorgung einer A2-Fraktur mit einem PFN. Der CCD-Winkel ist aufgerichtet, die Implantatlage korrekt. Die Antirotationsschraube lateralisiert bereits nach zwei Wochen (Abb.9) In der vierten postoperativen Woche kommt es zum Korrekturverlust, zum Cut-out der Schenkelhalsschraube und zur vollständigen Dislokation der Antirotationsschraube als Ausdruck der ungenügenden Stabilität nach der Erstoperation (Abb.10).

a

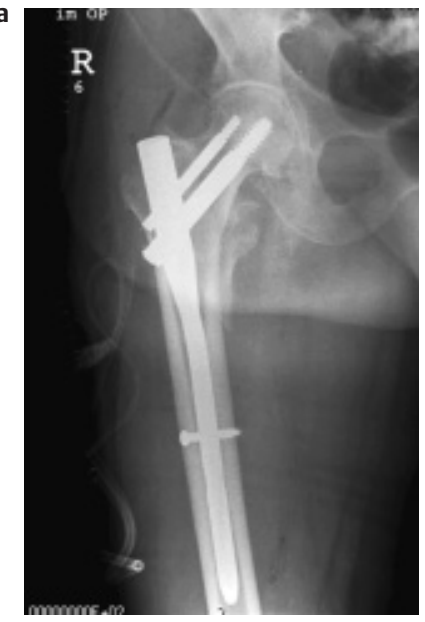

Korrekter CCD-Winkel und regelrechte Implantatlage allein garantieren nicht die Stabilität bei der Versorgung einer pertrochantären Femurfraktur vom Typ $31 \mathrm{~A} 2$ !

Im Sinne der Salvage Procedure erfolgte der Verfahrenswechsel auf ein winkelstabiles Implantat (LISS) (Abb.11). Ein Vorteil dieses Verfahrens liegt in der Möglichkeit der Operation in Seitenlage des Patienten.

Die Versorgung führte in diesem Fall nicht zu einer ausreichend stabilen Situation (Abb.12).

Das LISS hat sich in mehreren Fällen als Salvage Procedure nicht bewährt.

Ein weiterer Reosteosyntheseversuch (Winkelplatte) führte ebenfalls nicht zur Konsolidierung der Fraktur (Abb.13 und 14). Schließlich erfolgte der Verfah-

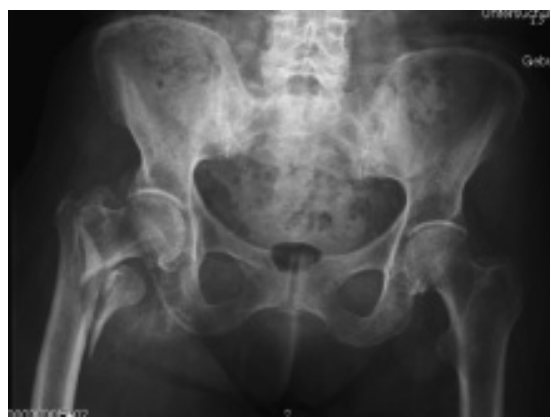

Abb.7 Beckenübersichtsaufnahme, 88-jährige Patientin nach Sturz im Heim, A2-Fraktur rechts.

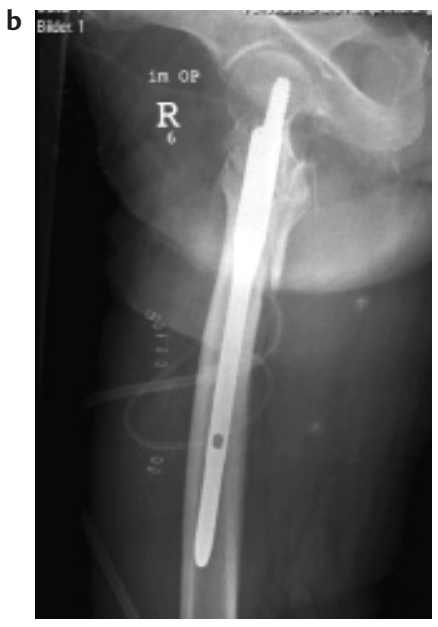

Abb. 8a, b A2-Fraktur mit einem PFN versorgt, Röntgenaufnahmen im Operationssaal. 


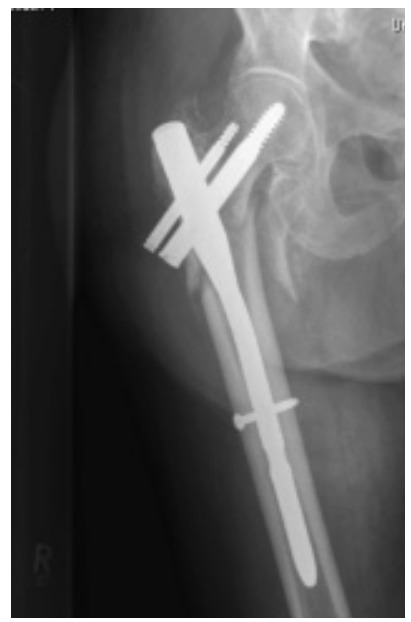

Abb. 9 Verlaufskontrolle 2 Wochen postoperativ, Antirotationsschraube lateralisiert.



Abb. 10 Verlaufskontrolle 4 Wochen postoperativ, Repositionsverlust und Implantatdislokation.

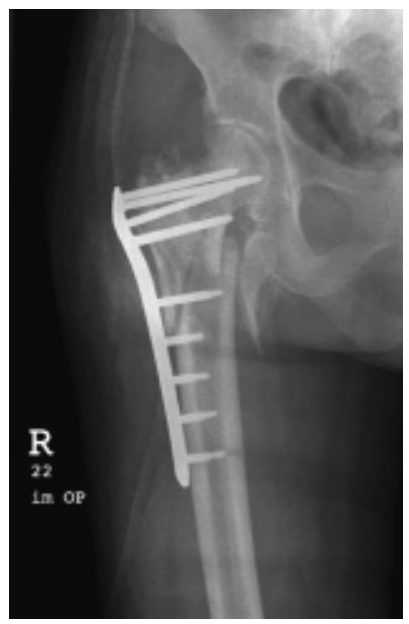

Abb.11 Verfahrenswechsel, LISS.

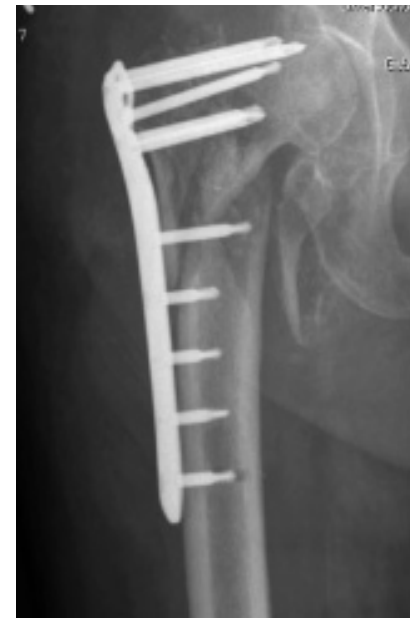

Abb.12 Redislokation nach der Stabilisierung mit dem LISS.

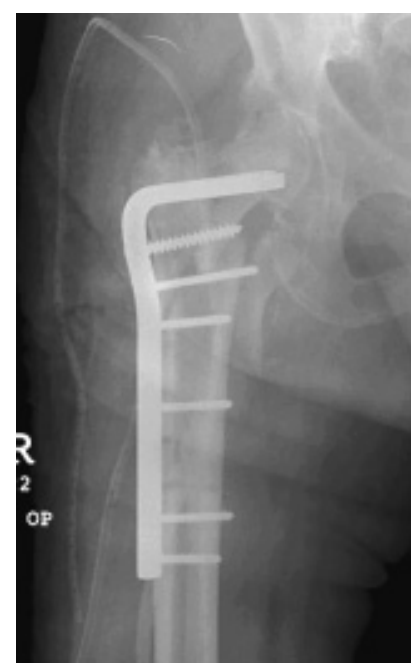

Abb.13 Verfahrenswechsel, Winkelplattenosteosynthese.

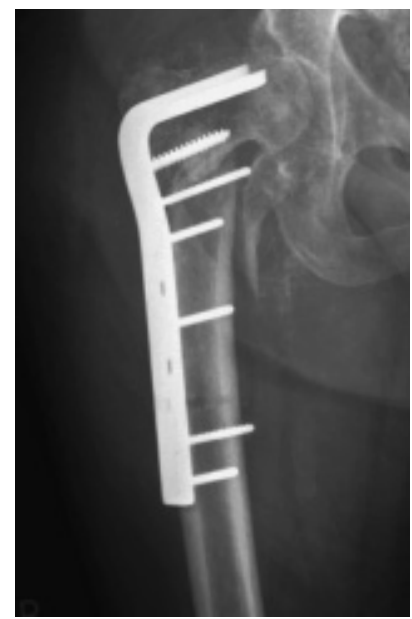

Abb.14 Erneuter Korrekturverlust.

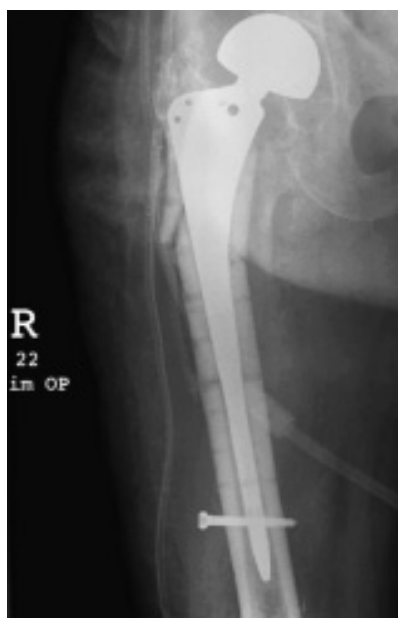

Abb.15 Implantation einer Duokopfprothese mit Revisionsschaft.

renswechsel zur Duokopfprothese (Abb. 15). Eine große Zahl der multimorbiden alten Patienten toleriert eine solche Vielzahl belastender Operationen nicht [10]. Diese Patientin verstarb einen Monat nach der endoprothetischen Versorgung in der Häuslichkeit.

Die primäre endoprothetische Versorgung bei instabilen pertrochantären Frakturen stellt nicht die einzige Alternative dar [5].

Es gilt bei der Primäroperation eine möglichst anatomische Reposition und Stabilisierung des Adam'schen Bogens zu erzielen. Dazu eignet sich eine Cerclage zur Reposition und Retention des Trochantor-minor-Fragmentes.

Folgende Schritte gilt es auch unter Bedingungen der Notfallversorgung einzuhalten:

- Genaue Frakturanalyse anhand der Röntgenaufnahmen

- Legen einer Tibiakopfdrahtextension, auch wenn die Operation am Unfalltag erfolgt. So wird eine dosierte und gleichmäßige Traktion intraoperativ gewährleistet.

- Nochmalige Frakturanalyse nach der Reposition auf dem Extensionstisch, ggf. Änderung des geplanten Verfahrens

- Korrekte Reposition, auch axial, und Aufrichtung des CCD-Winkels

- Maximale Stabilisierung des Adamschen Bogens, ggf. Drahtcerclage zur Retention des Trochantor minor 



Abb.17 Intraoperative Bildwandleraufnahme, korrekt liegender PFN, Adam'scher Bogen instabil.

Die Abb.16-18 zeigen eine instabile pertrochantäre Femurfraktur, den intraoperativen Befund nach Einbringen des PFN sowie nach zusätzlicher Stabilisierung des Adam‘schen Bogens.

Hinweise zur Erzielung einer ausreichenden Stabilität bei der primären Osteosynthese:

- Tibiakopfdrahtextension auch bei sofortiger Operation

- Frakturanalyse nach der Reposition auf dem Extensionstisch

- Stabilität des Adam'schen Bogens beurteilen

- Drahtcerclage zur Reposition und Retention der trochantären und subtrochantären Region
Abb. 16a, b Instabile pertrochantäre Fraktur, Unfallaufnahmen einer 93jährigen Patientin.

\section{Periimplantäre Frakturen}

Der PFN in der Standard-Ausführung (240 mm Länge) ist für das rechte und linke Femur anwendbar, das heißt, er berücksichtigt die Antekurvation des Femurs nicht. Mit dem Ziel, die Schenkelhalsschraube dicht über dem Adam'schen Bogen zu platzieren, wird der Nagel gelegentlich mit dem Hammer eingeschlagen [9]. Davor ist dringend zu warnen. Es kommt zu hohen Spannungen an der ventralen Femurkortikalis in Höhe der Nagelspitze, die zusammen mit der Bohrung für den distalen Verriegelungsbolzen dann den Ausgangspunkt einer Fissur bilden.

Die folgenden Abbildungen illustrieren einen solchen Kasus. Für die exakte Nagelplatzierung wurde der Hammer eingesetzt. Die postoperativen Röntgenaufnahmen im Operationssaal sind nicht ausreichend gewürdigt worden. Eine Fissur wurde nicht erkannt, erst als es im Verlauf zur periimplantären Fraktur kam. Es erfolgte der Verfahrenswechsel zum langen PFN (Abb.19-21).

Lässt sich der Nagel nicht in der gewünschten Position platzieren, wechselt man besser auf einen langen PFN, der die Femurantekurvation berücksichtigt.
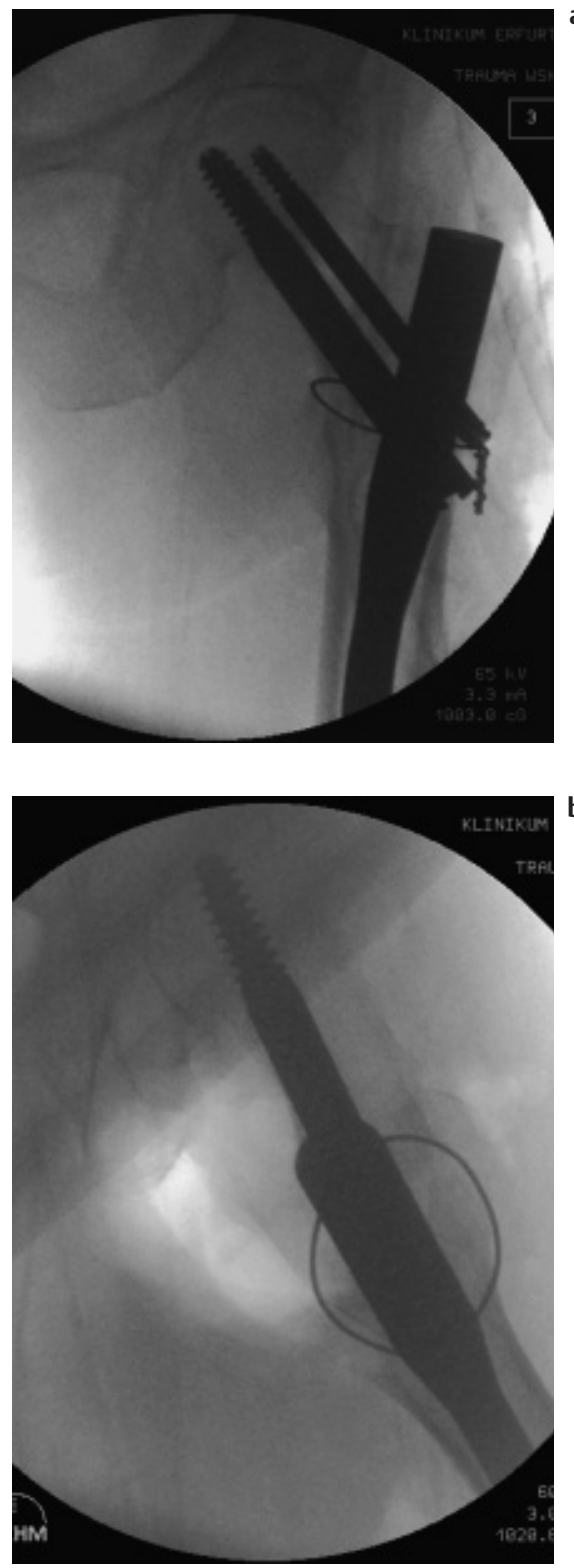

Abb.18a,b Erhöhung der Stabilität durch Drahtcerclage, intraoperative Bildwandleraufnahmen.

Prophylaxe periimplantärer Frakturen:

- Hammerschläge zur Positionierung des PFN sind zu vermeiden

- Besser auf einen langen PFN wechseln, Antekurvation dann berücksichtigt

- Verriegelungsbolzen nicht fest anziehen

- Postoperative Röntgenkontrolle unmittelbar im OP-Saal veranlassen und genau analysieren 

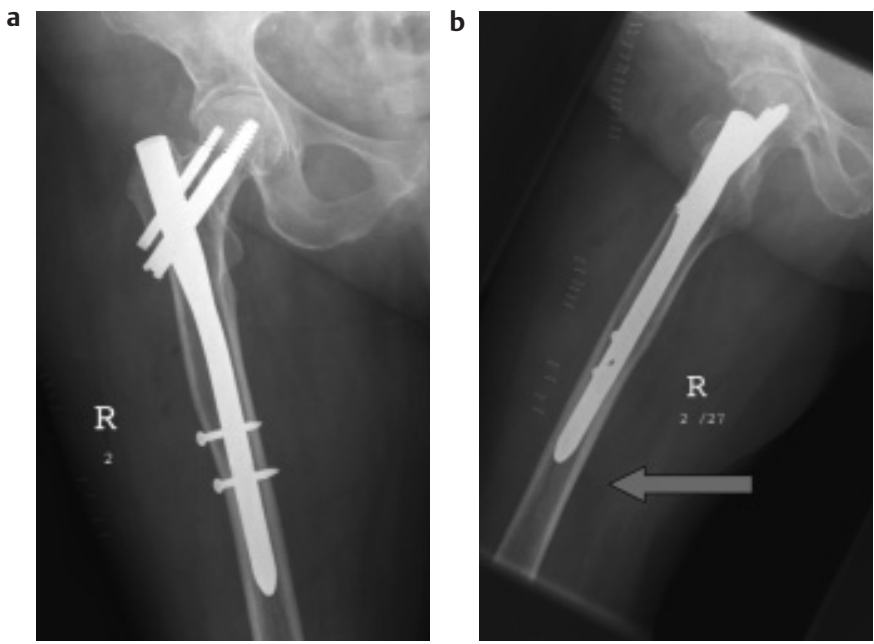

Abb.19a, b Versorgung einer A2-Fraktur bei einer 93-jährigen Patientin; nach Einschlagen des PFN lässt bei regelrechter a.-p. Projektion die axiale Röntgenaufnahme im Operationssal eine Fissur an der Nagelspitze erkennen.
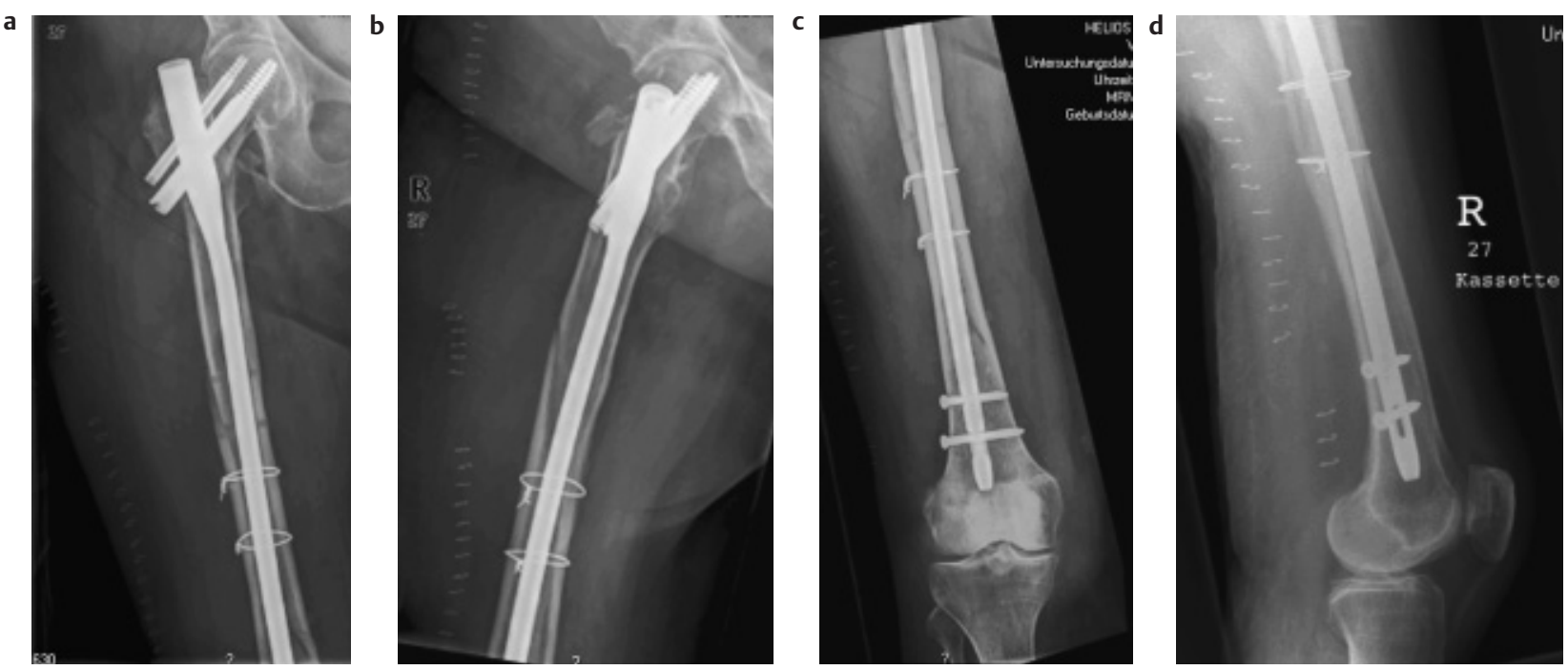

Abb. 21 a-d Röntgenaufnahmen nach dem Verfahrenswechsel im OP-Saal, langer PFN.

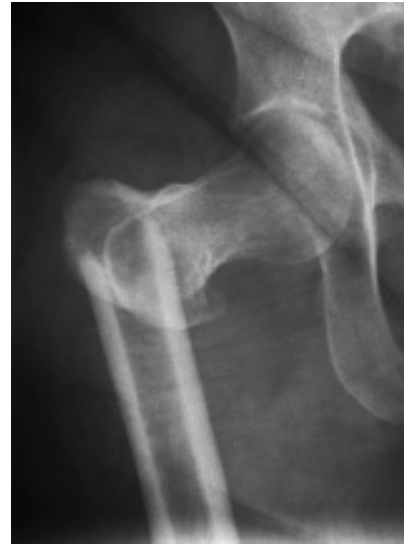

Abb. 22 Subtrochantäre Femurfraktur eines 71-jährigen Patienten, Unfallaufnahme.

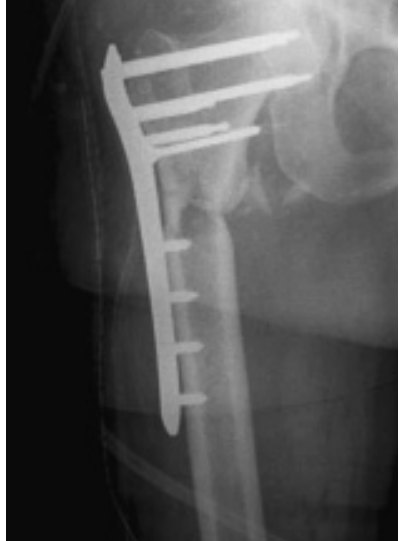

Abb. 23 Versorgung mit dem LISS.

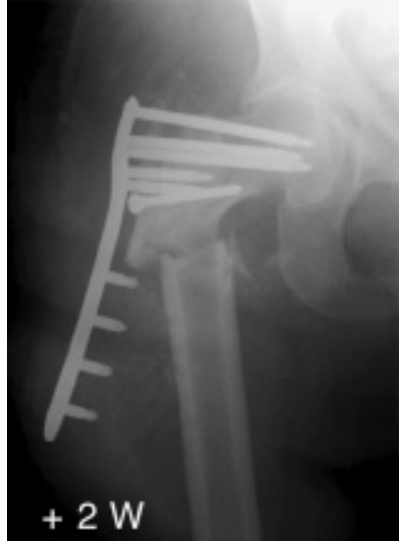

Abb. 24 Ausriss des LISS aus dem Femurschaft nach monokortikaler Montage.

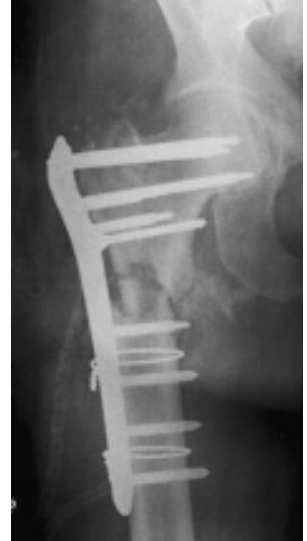

Abb. 25 Stabilisierung durch bikortkale Fixation und Cerclagen. 


\section{Subtrochantäre Femurfrakturen}

Subtrochantäre Femurfrakturen sind hochgradig instabile Verletzungen. Sie betreffen gehäuft auch jüngere Patienten im Rahmen von Hochrasanztraumen.

Die exakte Reposition gestaltet sich aufgrund des Zuges der Glutealmuskulatur erfahrungsgemäß schwierig.

Die Abb.22 zeigt eine subtrochantäre Fraktur mit der charakteristischen Fragmentdislokation.

Die primäre Stabilisierung nach exakter Reposition erfolgte mittels LISS (Abb. 23).

Es kommt zum Ausriss des LISS aus dem Femurschaft nach monokortikaler Montage (Abb.24). Ein Reeingriff führte zunächst $z u$ einer stabilen Situation (Abb.25) bis es zum Ausbruch der Schrauben aus dem proximalen Fragment kommt (Abb. 26 und 27). Die Lösung bestand in diesem Fall letztlich im Verfahrenswechsel zur Duokopfprothese mit Revisionsschaft (Abb. 28).

Die fehlgeschlagene Osteosynthese einer subtrochantären Femurfraktur endet nicht zwangsweise im endoprothetischen Ersatz.

Der folgende Kasus soll illustrieren, dass auch nach einem komplizierten Verlauf die knöcherne Ausheilung möglich ist.

Eine subtrochantäre Femurfraktur im Rahmen eines Polytraumas wurde nach initialer Retention im Fixateur externe (Abb. 29) mit einem langen PFN versorgt. Die postoperative Röntgenaufnahme im a.-p. Strahlengang (Abb.30) zeigt ein tolerables Ergebnis.

Die axiale Röntgenaufnahme dokumentiert hingegen die Fehlimplantation des Nagels (Abb. 31).

Kompliziert wurde der Verlauf durch eine mehrfach transfusionsbedürftige Nachblutung. Als Ursache konnte eine Leckage der Arteria femoralis superficialis verifiziert werden (Abb.32). Nach radiologischer Intervention (Stent-Einlage) war diese Komplikation beherrscht (Abb. 33).

Im Sinne der Salvage Procedure konnte der Verfahrenswechsel zur LISS-Montage, kombiniert mit Cerclagen, erfolgen.

Den Erfolg dokumentieren die Ausheilungsbilder nach 20 Monaten (Abb.34).

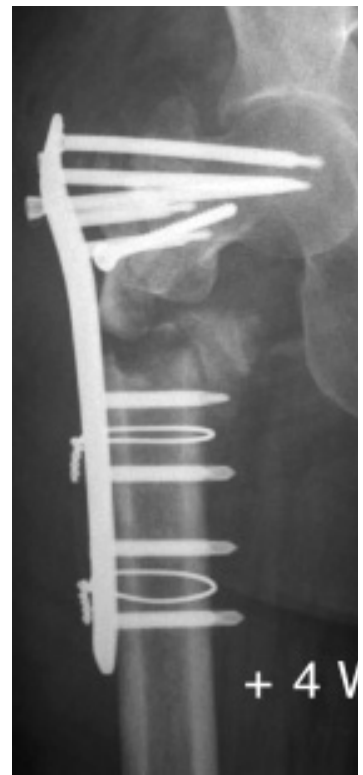

Abb. 26 Redislokation durch Ausriss des LISS aus dem proximalen Fragment.

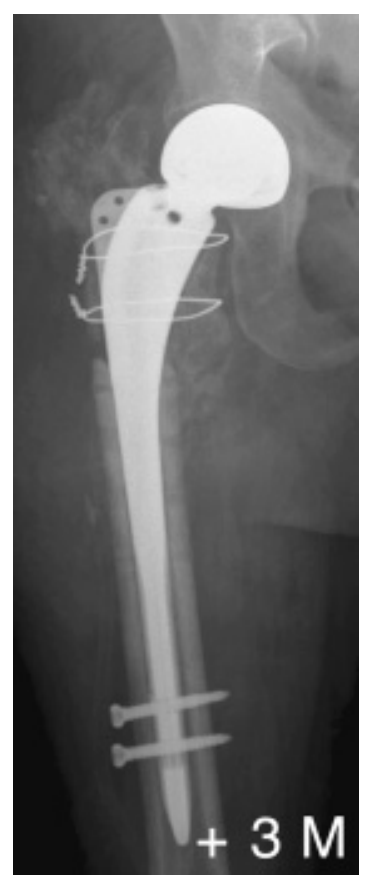

Abb. 28 Verfahrenswechsel, Implantation einer Duokopfprothese mit Revisionsschaft.

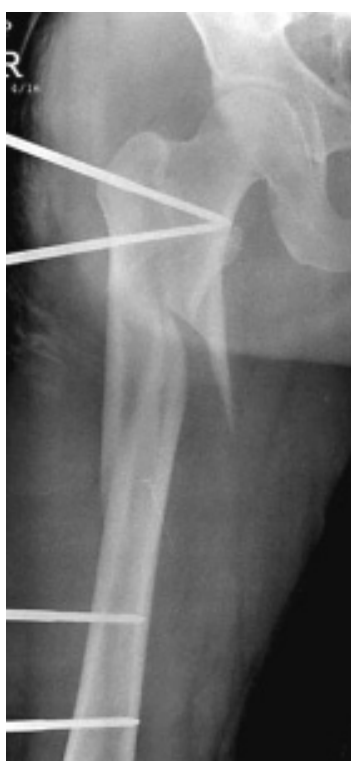

Abb.29 Subtrochantäre Femurmehrfragmentfraktur im Rahmen eines Polytraumas, initiale Retention im Fixateur externe, 55-jähriger Patient.

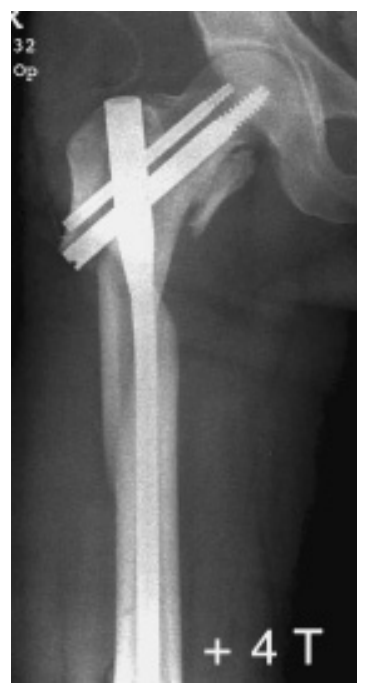

Abb.30 Versorgung mit dem langen PFN, postoperative Röntgenaufnahme a.-p. 


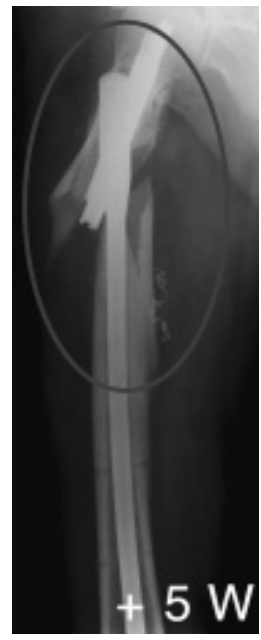

Abb. 31 Implantatfehllage, dokumentiert in der axialen Röntgenaufnahme.

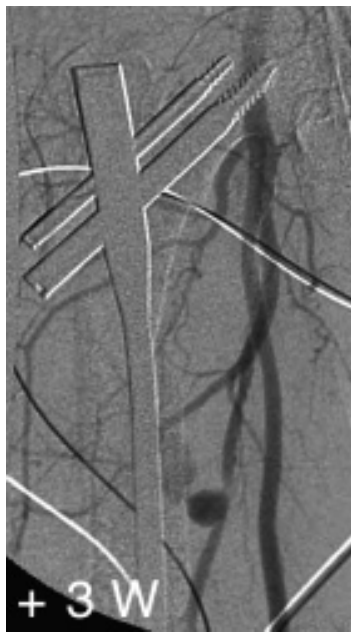

Abb.32 Angiographie wegen massiver Nachblutung: Leckage der A. fem. sup.
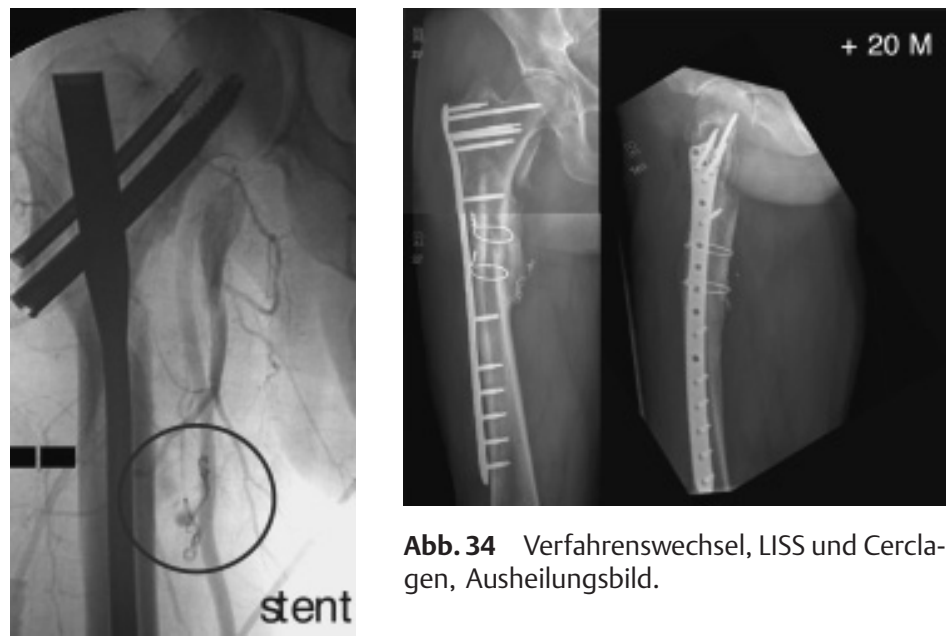

Abb. 34 Verfahrenswechsel, LISS und Cerclagen, Ausheilungsbild.
Abb. 33 Radiologische Intervention: Blutstillung durch Stent-Einlage.

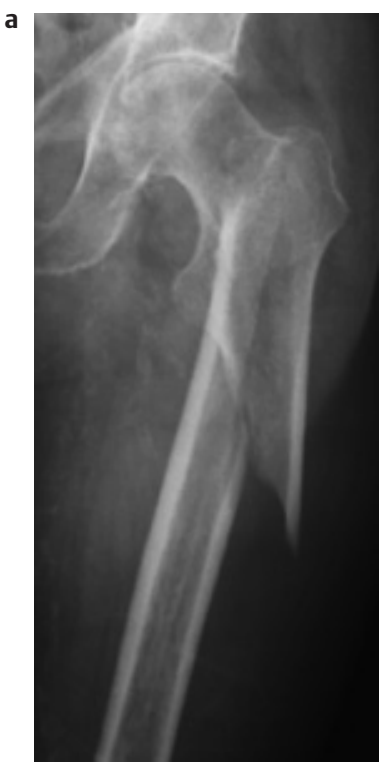

b

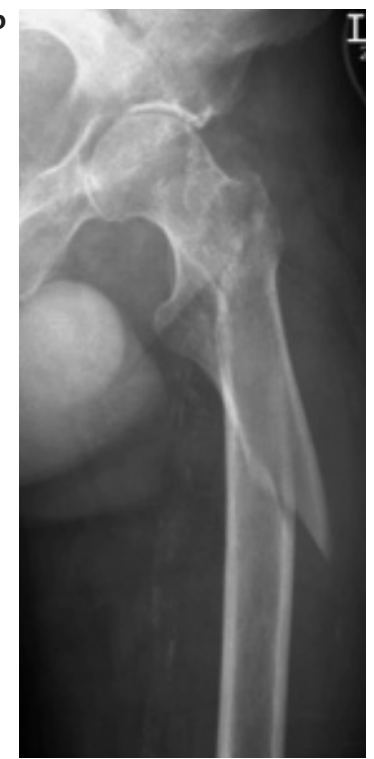

Abb. 35 a,b Subtrochantäre Femurfraktur links, Unfallaufnahmen, 86-jähriger Patient.
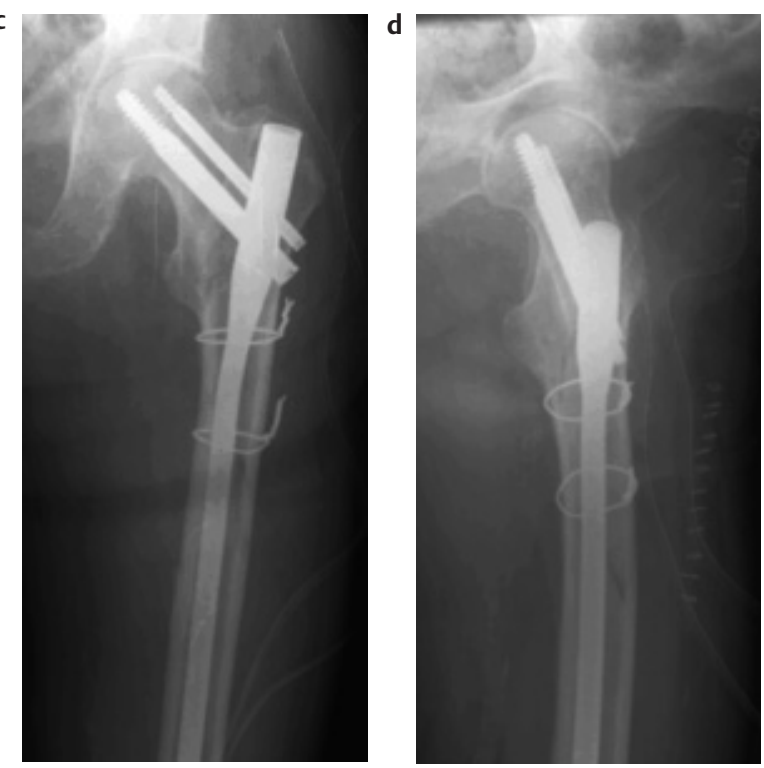

Abb. 35 c, d Stabilisierung der Fraktur mittels zweier Drahtcerclagen und langem PFN.
Der Verfahrenswechsel nach fehlgeschlagener Osteosynthese muss nicht zwangsläufig in den prothetischen Ersatz münden!

Für die initiale Stabilisierung subtrochantärer Femurfrakturen empfiehlt sich folgendes Prozedere:

- Reposition und Retention mittels zweier Drahtcerclagen

- Anschließend Einbringen des intramedullären Kraftträgers (Abb.35a-d)
Bei subtrochantären Femurfrakturen Reposition und Retention mittels Drahtcerclagen vor dem Einbringen des Nagels!

\section{Schlussfolgerung}

Die hohe Inzidenz der per- und subtrochantären Femurfrakturen darf nicht zu einer Bagatellisierung ihrer Versorgung führen.

Die fehlgeschlagene Osteosynthese stellt ein sehr ernsthaftes Problem dar und ist häufig auf eine postoperativ ungenügende Primärstabilität zurückzuführen.

\section{Literatur}

${ }^{1}$ Ahrengart L, Törnkvist $\mathrm{H}$, Fornander $\mathrm{P}$, Thorngren KG, Pasanen L, Wahlström $\mathrm{P}$, Honkonen S, Lindgren U. A Randomized Study of the Compression Hip Screw and Gamma Nail in 426 Fractures. Clin Orthop 2002;

Volume 1 (401): 209-222

${ }^{2}$ Doering R, Winker KH. Die pertrochantäre Femurfraktur. OP-Journal 2002; 17: 128 131

${ }^{3}$ Hoffmann R, Schmidmaier G, Schulz R, Schütz M, Südkamp, NP. Classic nail versus DHS. A prospective randomised study on operative fixation of trochanteric femur fractures. Der Unfallchirurg 1999; 102: $182-190$ 
${ }^{4}$ Josten Ch, Tiemann AH. Komplikationen nach proximalen Femurfrakturen. OP-Journal 2002; 17: 148-153

${ }^{5}$ Perka C, Ludwig S, Stern S. Results of total Hip Arthroplasty after Failed Internal Fixation of Hip Fractures. Z Orthop 2000; 39-45

${ }^{6}$ Robinson CM, Adams CI, Craig M, Doward W, Clarke MCC. Implantat-Related Fractures of the Femur Following Hip Fracture Surgery, JBJS. ORG 2002; Volume 84-A, Number 7: 1116 - 1122

${ }^{7}$ Valverde J, Alonso M, Porro J, Rueda D, Larrauri P, Soler J. Us of the Gamma Nail in the Treatment of Fractures of the Proximal Femur, Clin. Orthop, 1998, Volume 1 (350): $56-61$
${ }^{8}$ Wagner R, Blattert TR, Weckbach A. Solution for Extraarticular Hip Fractures Using the "Sliding-Srew-Nail“-Principe. Results of Two Different Systems (Classic Nail an Gamma Nail). Der Unfallchirurg 1998; 101: $894-$ 900

${ }^{9}$ Werner-Tutschku W, Lajtai G, Schmiedhuber G, Pirkl C, Orthner E. Intra- und perioperative Komplikationen bei der Stabilisierung von per- und subtrochantären Femurfrakturen mittels PFN. Der Unfallchirurg 2002; 105: $881-885$

${ }^{10}$ Zuckermann J, Skovron ML, Koval K, Aharonoff G, Frankel V. Postoperative Complications and Mortality Associated with Operative Delay in Older Patients Who Have a Fracture of the Hip. JBJS Am 1995; Volume 77-A(10): $1551-1556$
Dr. med. Andreas Luther

Oberarzt

Dr. med. Steffen Langenhan

Oberarzt

Prof. Dr. med. Karl Heinrich Winker

Chefarzt

Klinik für Unfall-, Hand- und

Wiederherstellungschirurgie

HELIOS Klinikum Erfurt

Nordhäuser Str. 74

D-99089 Erfurt

khwinker@erfurt.helios-kliniken.de 